\title{
Operating Nomad during the Atacama Desert Trek
}

\author{
David Wettergreen, Maria Bualat, Daniel Christian, Kurt Schwehr, \\ Hans Thomas, Deanne Tucker, and Eric Zbinden \\ Intelligent Mechanisms Group \\ NASA Ames Research Center, MS 269-3 \\ Moffett Field, CA 94035-1000 USA
}

\begin{abstract}
Nomad is a mobile robot designed for extended planetary exploration. In June and July of 1997, Nomad performed the first such mission, traversing more than 220 kilometers in the Atacama Desert of Chile and exploring a landscape analogous to that of the Moon and Mars. Nomad's journey, the Atacama Desert Trek, was an unprecedented demonstration of long-distance, long-duration robotic operation. Guided by operators thousands of kilometers away but telepresent via immersive imagery and interfaces, Nomad operated continuously for 45 days. Science field experiments evaluated exploration strategies and analysis techniques for future terrestrial and planetary missions.
\end{abstract}

\section{Introduction}

In June 1997, the mobile robot Nomad was deployed to the Atacama Desert of Chile (Figure 1). In 45 days Nomad traveled an unprecedented 220 kilometers through rough terrain while controlled by operators thousands of kilometers away. This activity, the Atacama Desert Trek, tested technologies critical to terrestrial and planetary field operations and may prove to be a milestone in robotic exploration. The Nomad project addressed issues of robotic configuration, communications, control, visualization and remote science.

The Nomad robot was developed by researchers from the Robotics Institute at Carnegie Mellon University. In the Intelligent Mechanisms Group at NASA Ames Research Center, we focus on supervised autonomy and develop robot architectures and operator interfaces appropriate to this type of telerobotic control. Our research is grounded in testing real robots in real field experiments with real planetary scientists. NASA Ames partnered with Carnegie Mellon to develop Nomad's operator interface including a "virtual dashboard" and visualization of Nomad's unique imagery.

During the desert trek, we organized science field experiments intended to test remote geological investigation, paving the way for new exploration strategies on Earth and beyond.

In this paper we will overview the Nomad project and the robot, and then focus on the development of the operator interface for the Nomad robot, describe the details of how

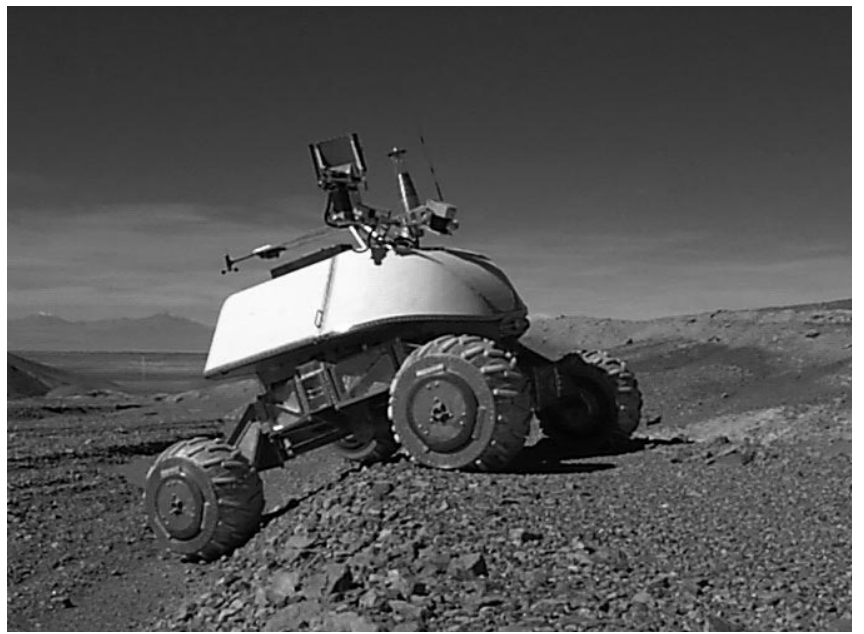

Figure 1: Nomad in the Atacama Desert of Chile

Nomad is teleoperated, and discuss the results of field experiments conducted as part of the Atacama Desert Trek.

\subsection{Objectives}

\section{Long-distance, long-duration exploration}

The primary objective of the Nomad project was to develop, demonstrate, and evaluate a robotic system capable of longdistance, long-duration planetary exploration. Nomad's goal was to navigate 200 kilometers across the rugged Atacama Desert in South America while under the control of operators in North America.

\section{Simple, compelling interface}

By combining panoramic visualization with an easily comprehensible operator interface, the desert trek provided an interactive experience that we believe holds great promise for remotely-guided geologic exploration. It also provided the general public a compelling interactive experience and an opportunity to remotely drive an exploratory robot.

Remote geologic interpretation and mission simulation A series of field experiments conducted during the desert trek simulated robotic operation on other planets with methods and technologies relevant to near-term planetary missions. These field experiments serve as a test for remote geological 
investigation, and validate new strategies for terrestrial and planetary exploration.

\section{Nomad Robot}

The Nomad mechanism features four-wheel drive, fourwheel steering on a transforming chassis. It carries a panospheric camera with a 360 degree field-of-view to provide continuous imagery of the surrounding terrain. To support high-bandwidth telemetry, Nomad uses an actively pointed antenna. A detailed description of Nomad is available in [Whittaker, 1997].

\subsection{Four-wheel drive and steering in a transforming chassis}

The robot's four-wheel drive, four-wheel steering locomotion and innovative suspension system provide effective traction, mobility, and propulsion across loose sands, rocks and soils. The aluminum wheels have cleats along the circumference and motors and gears enclosed inside.

The 725 kilogram mechanism's chassis expands to 2.4-by2.4 meters to improve stability and propulsion over variable terrain and collapses to 1.9-by-1.9 meters for stowage, shown in Figure 2. Nomad steers by adjusting a pair of four-
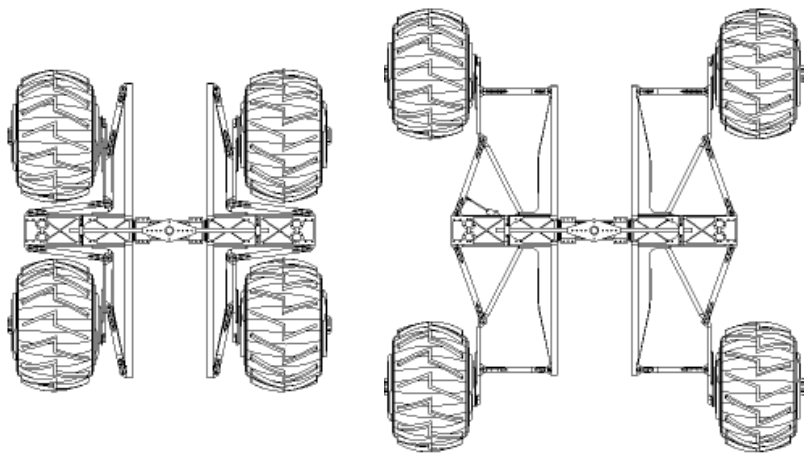

Figure 2: Nomad chassis stowed (left) and deployed (right) showing steering linkages and bogey-pivot axis

bar mechanisms on either side of the chassis. An averaging linkage between the left and right sides guides Nomad's body shell on a smooth path between the extremes of motion experienced by the wheels. The body shell encloses all computing, electronics, and sensors including an inertial measurement unit and gyrocompass.

Nomad is powered by a 3.5 kilowatt gasoline generator, not applicable to planetary exploration but necessary for this experimental system. Nomad is a prototype vehicle and many issues must still be addressed to prepare it for future missions.

\subsection{Panospheric imaging of complete surroundings}

Immersive imagery is often the key to effective teleoperation. Traditional cameras provide limited field-of-view but Nomad carries a panospheric camera that generates imagery with an ultrawide field of view, shown in Figure 3.n

The camera captures a 360 degree view of the surrounding terrain (to 40 degrees above horizontal) using a spherical

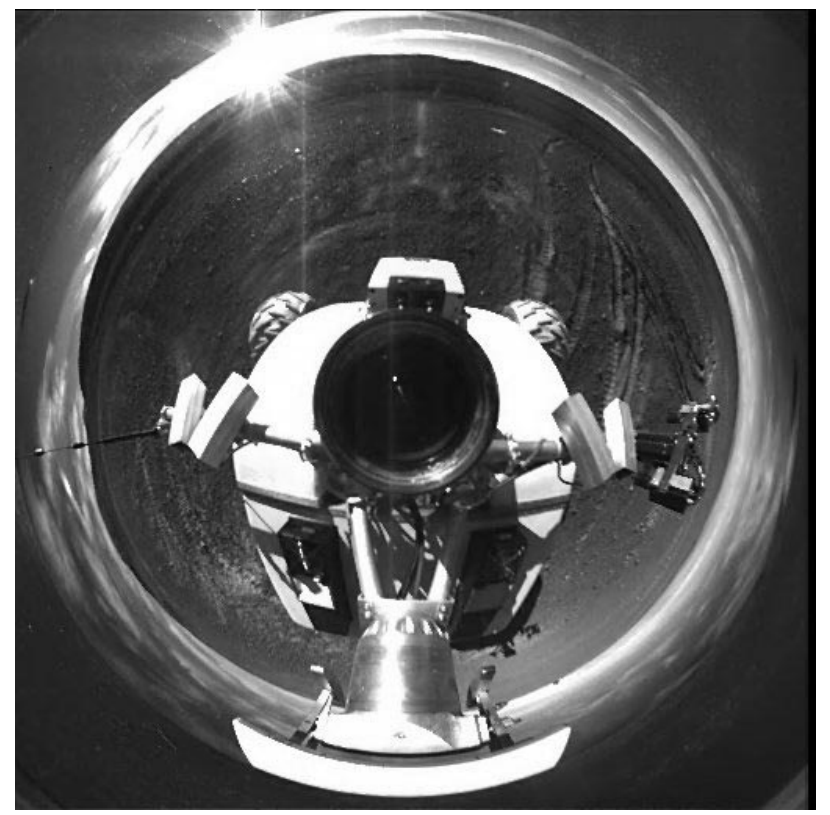

Figure 3: Image from the Panospheric Camera

mirror mounted above of a vertically oriented digital camera. The mirror is a $70 \mathrm{~mm}$ plano-convex lens with a $60 \mathrm{~mm}$ diameter chord, so the mirror is less than a hemisphere. The scene is imaged with a $1024 \times 1024$ color CCD chip with $12 \mathrm{um}$ pitch and alternating color mask for an effective color resolution of $512 \times 512$. Images are read out in vertical columns at approximately $6 \mathrm{~Hz}$ and shifted from the digital camera via a high-speed frame grabber to a dual-processor Pentium Pro 200MHz computer running the Windows NT operating system.

Each image is processed by a commercial compression package that applies a wavelet-based compression algorithm to reduce images from 786 kilobytes $(512 \times 512 \times 24$ bits) to a more manageable size for network transfer.[Summus, 1997] The resulting compressed images are about 30-60 kilobytes using compression of 15-30:1. One CPU is dedicated to compressing images at a rate of $2.5 \mathrm{~Hz}$.

The compression processor queues images into shared memory where a messaging process implemented with NDDS, a commercial UDP-based messaging package, [RTI, 1997] breaks each image into 8 kilobyte packets. These packets are multicast from Nomad and received in multiple locations. Each packet contains a packet identification code and the total number of packets in the image, and a variable length data block. This queuing system allows the image capture and compression system to be decoupled from the delay of sending the images out on the network. The delay per packet was originally calculated based on the known available bandwidth however, the delay was adjusted to minimize the number of lost packets. During the Atacama Desert Trek, more than one million panospheric images were transmitted at approximately $1 \mathrm{~Hz}$.

\subsection{Communication via actively-pointed antenna}

Nomad aims its directional antenna to achieve data rates higher than usually attained from a moving platform. Mobile 
robots typically use omnidirectional antennas for simplicity but are limited in data rate and range of communication. Nomad achieves high data-rate communication (over $1.5 \mathrm{Mbps}$ ) over extended range (from 5 to 10 kilometers) by actively pointing its high-gain directional antenna.

Communication from the robot is performed through a high-speed wireless ethernet system operating on the $2.4 \mathrm{Ghz}$ spread spectrum band. To get the required range and bandwidth, highly directional antennas are used on both ends of the link. If either antenna does not track accurately or if there is an obstruction in the line-of-sight between the antennas, then communications is lost until the situation is remedied. A separate low-bandwidth radio system provides communication without pointing or line-of-sight requirements to allow the robot to recover from interruptions in the high bandwidth link.

\section{Virtual Dashboard Interface}

The Intelligent Mechanisms group created an operator interface, called the Virtual Dashboard (Figure 4), for driving Nomad. Our intention is always to develop operator interfaces that are simple and intuitive to use, provide compelling interaction with the remote robot explorer, and result in more efficient and effective science operations. Our objectives are to: simplify assessment of current robot state; reduce the number of operators and required skill level; develop accurate understanding of robot's environment; and operate as effectively telepresent, as if the operator were physically present with the robot.

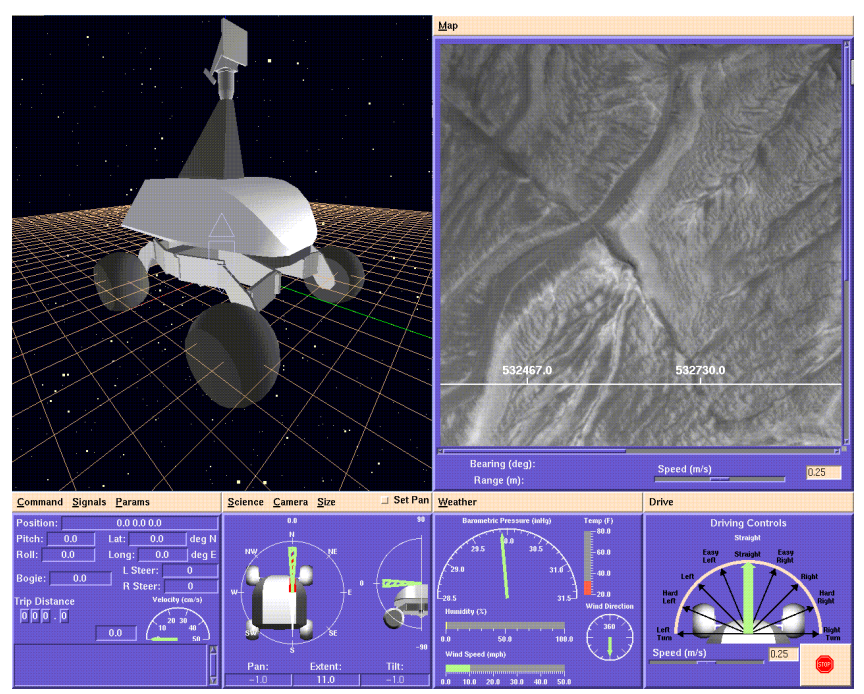

Figure 4: Virtual Dashboard

The Virtual Dashboard provides a clear visualization of the robot's state in recognizable graphical and numeric formats. The robot's position is plotted on aerial images, and the pose is rendered in 3-D, all updated in real-time. An operator can quickly assess Nomad's condition and command it, using a mouse to click the direction to steer the robot or point the cameras. This improves efficiency and results in more rapid site exploration.

The Virtual Dashboard is written in the TCL/Tk inter- preted scripting language, which allows rapid development of using built-in widgets for buttons, dials, slides, etc. We developed a package that interfaces TCL to NDDS and enables the Virtual Dashboard to send commands and receive telemetry to and from the systems on-board Nomad.

The Virtual Dashboard is made up of multiple, reconfigurable panels that provide graphic telemetry displays and robot control widgets. In all panels, the operator may use menus to reconfigure panels or bring up interface help files.

\subsection{Telemetry display panel}

The telemetry panel displays position, pose and state information for the operator. Several textual displays show Nomad's position in world coordinates, the pitch and roll angles, latitude and longitude, chassis bogey angles and turn radius. A trip distance counter tracks the distance travelled since the last counter reset. The speedometer dial shows Nomad's speed in the inertial reference frame as given by the velocity output of the GPS system.

The telemetry panel contains a message window in which notices of Virtual Dashboard activity and warnings about approaching or exceeded preset limits are display (along with an audio alarm). For example, the Virtual Dashboard will warn the driver that an excessive pitch or roll value has been detected, or that the generator is low on fuel.

Through the use of the telemetry panel menu options, the operator can deploy or stow the wheels, reset the trip counter, make log entries, or change servo or broadcast parameters.

\subsection{Aerial tracking panel}

The aerial tracking panel displays aerial photos of the Atacama desert with Nomad's position indicated in the terrain. It provides controls for sending heading and waypoint commands. The panel also contains a display of the commanded bearing and range and the speed control.

Each aerial image comes with a set of equations that are used to determine the pixel location within the image of a given pair of coordinates. The output of Nomad's GPS locates it within the current image. As it moves, the dashboard puts down a marker at each location so that the rover's path is traced out on the image. As it nears the edge of the current image, the dashboard loads a neighboring image.

The operator creates bearing and waypoint commands using mouse clicks and drags within the aerial image. Menu options allow the operator to send the bearing and waypoint commands, or to reset the navigation system.

\subsection{Camera control panel}

The science camera panel displays rover compass data and contains the controls for the science imaging package onboard Nomad. The science package is made up of a pair of high resolution color cameras and a pair of wide-angle monochromatic cameras mounted on a pan-tilt mechanism.

The camera/compass display takes up the left two thirds of the panel while the tilt display uses the right third. The compass rotates to reflect the incoming telemetry. The camera controls allow the operator to maneuver the pan-tilt device and to set the mosaic pan and tilt ranges using simple mouse 
clicks and drags.

By overlaying the pan controls on top of the compass display, we simplified control of the pan angle. Typically, a geologist will request an image from an absolute bearing. With the rover bearing display and the relative pan angle display co-located, there is no need to translate from absolute directions to relative pan-angles. The operator may use the menu options to display images taken by the science cameras, grab images or sequences of images, set camera parameters, or home and park the pan-tilt device.

\subsection{Driving control panel}

The driving control panel contains the controls for sending direct teleoperation commands to the rover. The operator sets the turn radius by rotating a radial dial and sets the speed and distance controls with slider bars (or keyboard input). Once all the parameters of the drive command have been set, the user need simply click in the panel to send the command. An emergency stop button immediately sends a low-level command to Nomad to shut-down servos.

The Virtual Dashboard binds the arrow keys on the keyboard to drive commands. The operator can adjust the rover's speed by pressing the up and down arrows, and can adjust the turn radius by pressing the left and right arrows. Novice drivers tended to use the visual information from the panospheric camera as their primary source of driving feedback, only occasionally glancing at the dashboard for roll and pitch information. They found the keyboard bindings, in particular, extremely useful because it allowed them to send commands without having to look away from the panospheric imagery. Because of their greater dependence on the panospheric display, which carried with it a 5 second latency, novice drivers also tended to over-steer the vehicle when driving at top speeds. Experienced drivers made greater use of the compass, position, and pitch and roll telemetry displays, but also found the keyboard bindings useful as they allowed quick command adjustments to be sent.

The Virtual Dashboard can be configured to display a number of additional panels including individual wheel control, antenna pointing, and weather information. Experienced drivers developed their own preferred configuration for the Virtual Dashboard.

The Virtual Dashboard incorporates a rendered 3-D model of Nomad with each articulation updated from live telemetry at $5 \mathrm{~Hz}$. This provides an accurate representation of Nomad's relative position, orientation, and kinematics, which can be viewed from any perspective. This virtual environment model was a central component of previous rover interfaces [Piguet, 1995] but, interestingly, because Nomad could not provide a detailed local terrain model there was insufficient context to interpret the scene. Many operators chose not to view the virtual Nomad because they found it misleading without more complete information about its relationship to the surrounding terrain.

\section{Telepresence Interface}

The Telepresence Interface uses the encompassing imagery from Nomad's panospheric camera to create a sense of pres- ence in the robot's environment. When Nomad's imagery is dewarped and displayed, operators can look around, from Nomad's perspective, with smooth and natural motions, and with new images arriving every second. (Figure 5) This telepresent view is projected on a large screen in front of the operator providing an immersive forward-looking perspective that appears beyond the Virtual Dashboard, like the windshield beyond the dashboard of a car.

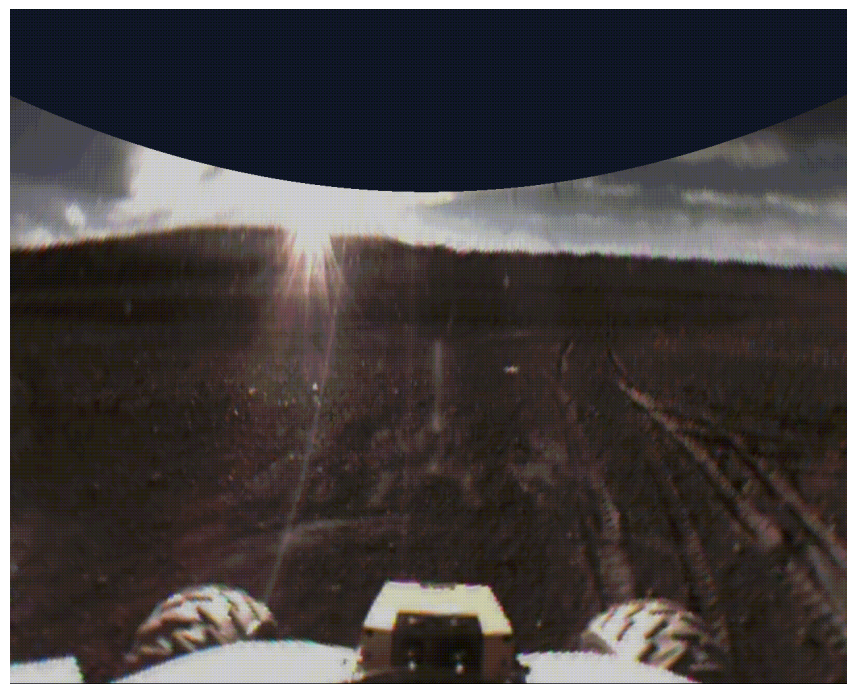

Figure 5: Display of panospheric imagery (from Figure 3)

We have integrated software to take an image, waveletcompress it, decompose it into packets, transmit these to multiple remote computers, reconstruct and finally decompress it into the original image. The Telepresence Interface was developed jointly with the GROK lab of University of Iowa and has several components: image transmit/receive, image compression/decompression, and image display. Image capture, compression and transmit, take place onboard Nomad as previously described. Image packets are received and reassembled at multiple remote viewing locations. The image is decompressed and then passed off to an SGI Performer application which texture maps the image onto the inside of a sphere. By mapping an image of a spherical mirror into a sphere and then placing the viewpoint at the center of that sphere, the image is intrinsically dewarped. Using an input device, like a 3-D mouse, an operator can spin around inside the sphere (with $30 \mathrm{~Hz}$ update) and view the world around Nomad in any direction.

The Telepresence Interface runs on a number of different multi-processor SGI graphics platforms-at least two processors are required to concurrently receive, decompress, and display images. Our target update rate was $7.5 \mathrm{~Hz}$ however, to date, the average is approximately 1 image per second ( 2 maximum) with a latency of approximately 5 seconds. The rate is limited by the speed that image packets can be queued and transmitted across the network. The latency is introduced by the time required to compress and decompress each image.

Through the month and a half of the Atacama Desert Trek, the Virtual Dashboard and Telepresence interfaces were used 
as the primary driving interface at both NASA Ames and the Carnegie Science Center in Pittsburgh.

During the science field experiments, experienced drivers at NASA Ames viewed the Telepresence Interface on a large projection screen while sitting in front of a workstation running the Virtual Dashboard. They controlled Nomad's movements and teleoperated it along detailed paths designated by a team of planetary scientists.

At most other times, Nomad was driven by a novice driver, usually a member of the public, at the Carnegie Science Center. These operators were allowed to drive anywhere they wished. They viewed panospheric imagery in a theatre with three projectors illuminating a 4m-by-12m (220 degree) curved screen. Most of the 100 novice drivers found the controls easy to learn, and after a few minutes of explanation, were able to drive Nomad independently.

In all, Nomad's operator interfaces performed well for two types of driving situations: experienced drivers with specific duties to perform, and novice drivers exploring at random. Learning time was very short for both types of drivers.

\section{Web Interface}

As part of the Atacama Desert Trek we developed a web site (http://img.arc.nasa.gov/nomad) through which all data from the science experiments and much of the telemetry and imagery was available as soon as it was received. Ancillary information was also available for the science teams to assist in finding and processing the data and understanding the rover capabilities. Additionally, the web site provided public outreach on all aspects of the mission.

\subsection{Panospheric Images}

Panospheric images, in wavelet-compressed format, were received at a rate of one per second, and each hour one image was decompressed, dewarped and made available on the web site. At the end of each day, the compressed images were written to CDROM and removed from the web site to make space for the next day's images. In the five days of science field experiments, 46,269 files comprising 2.8 gigabytes were archived for scientists. Attempting to go back and find events was only manageable when we logged the time span of interest in advance. The entire Atacama Desert Trek produced more than 30 gigabytes which were stored on 54 CDROMs.

\subsection{Science Images}

The science images from the on-board stereo high resolution and wide angle cameras were made available immediately on the web site. We retained the original format as an archive, and converted to formats compatible with analysis tools and web browsers. We converted the science images to an annotated format with the header and telemetry information written into the image.

In total, 1645 science images were recorded (402 megabytes in total size) with most being high-resolution color stereo pairs for geologic analysis, and monochrome for mosaicing. Full- and half-scale images are typically considered useful for science analysis; lower resolutions are used to sight the high resolution cameras or for navigation.

\subsection{Telemetry-Image Correlation and Logging}

From our previous field experiments, we had determined the importance of correlating an image with information describing the conditions under which is recorded, for example the camera's orientation. We developed a method of quickly locating the temporally-related rover telemetry and operator $\log$ annotation for an image through a log/image correlator page on the web site. Users can search by image size, time, or type, and view the image and all related information. This was achieved by writing the telemetry logs in HTML with hyperlinks to the images. File size must be limited to reduce web browsers load times, so judicious selection and organization of data is important.

\subsection{Web-based Collaborative Exploration}

An objective in conducting simulated exploration missions is to train planetary scientists on the use of rovers and advanced data visualization and analysis tools.

We prepared briefing packets to answer questions about the robot, its capabilities, and exact specifications of its sensors. Also on the web, we compiled and presented background information, technical information, related links, and information and photographs on the Atacama desert. This reference information was before and during the experiments. One of the most popular web pages contained field logs, very informative, entertaining accounts of the experience, written by engineers in Chile. We received appreciative messages from people all over the world regarding the logs.

We have been designing our web pages under the assumption that scientists will wish to be able to access mission data remotely. Certainly it is possible for scientists to access data via remote logon to project computers, however the web is ideally suited to easy data access independent of the underlying operating system and system architecture. The original data is always available in bulk, but the web allows for a more structured organization of the information, live updates, and easy access. The web interfaces are also be useful to access data throughout a mission-the continued popularity of the Mars Pathfinder site is evidence of this.

\section{Science Imaging System}

Our ongoing research has been developing tools that facilitate the imaging and analysis of the geology of remote regions. The Intelligent Mechanisms group's most recent remote science field experiment used monochrome stereo cameras for driving and 3D mapping and a monochrome camera with a filter wheel for color.[Christian, 1997] The stereo images were viewed with special glasses to see depth and the color images had to be assembled from the separate filter images by hand. The accuracy of the scientific analysis using this sensor and software suite was poor.

In order to determine if imaging resolution was limiting the scientific accuracy, the science imaging system for the Nomad field experiment was designed to match the resolution of the foveal region of the human eye. A pair of highresolution color cameras on a pan-tilt unit provide these capabilities. 


\subsection{Imaging camera and pointing device}

The science imaging system is designed to be very simple to integrate onto Nomad. The sensing head consists of a pan-tilt unit, the stereo jig, two color cameras, and two monochrome cameras. Shrouds protect the color cameras from rain and physical contact. The electronics box contains the computer, power supplies, and control electronics. Cabling from the sensing head connects to the electronics box cabling at the electronics enclosure bulkhead.

The stereo jig allows the two color cameras to be precisely aligned in pitch, roll, and vergence. Separate adjustment and lock down screws keep the jig in alignment even under the prolonged vibration from $200 \mathrm{~km}$ of driving. The rotation center for the tilt axis matches the camera imager. In order to reduce loading on the pan-tilt due to vehicle pitch, roll, and shock, the center of gravity of the stereo head is very close to the pan-tilt rotation center.

The smallest triple-CCD cameras, Sony XC-003s, with $1 / 3$ inch imagers and c-mount lenses, were selected. With a $25 \mathrm{~mm}$ lens, they provide 0.30 milliradian per pixel image resolution. This matches well with 0.25 to 0.33 milliradian resolution typical of the foveal region of the human eye. This high resolution results in a rather narrow field of view of $11^{\circ}$. To extend the field of view, mosaics of multiple images may be taken and then assembled into a larger image. The monochrome cameras provide a wider field-of-view $\left(33^{\circ}\right)$ for sighting the high resolution cameras.

The color cameras have a 10 inch baseline and are verged and focused at 5 meters. The cameras are mounted 1.5 meters above the ground. These settings yield the best resolution for objects on the ground about 3 meters in front of the robot. (See Figure 6.)
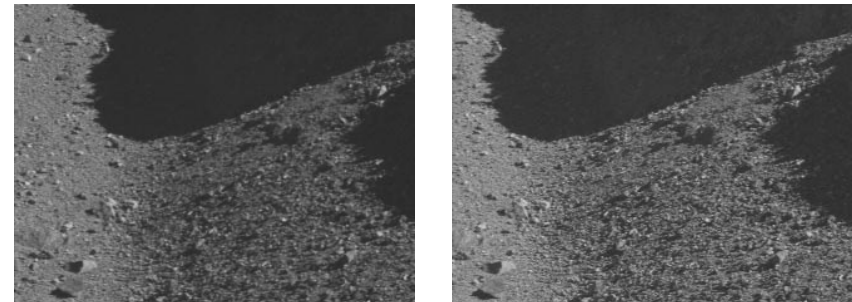

Figure 6: High-resolution monochrome stereo pair

The stereo jig is mounted to a pan-tilt unit chosen because of its payload capability, low weight, fast response, and servo-based controls. A servo unit was used to avoid losing positioning due to shocks to the robot.

In order to minimize errors due to vehicle vibration, the stereo cameras are synchronized to each other and digitized simultaneously. Two digitizers with the RGB option digitize the images and hold them in on-board memory. The two digitizers are mounted in the PCI bus of a ruggedized Linux computer system. A serial port controls the pan-tilt drivers and power supply. An ethernet card allows communication with the other on-board computers and the radio ethernet. The system boots from an on-board hard disk and starts the necessary daemons and the network interface.

Stereo image pairs are sent as a single image with the left image above the right image. Mosaics are sent as a sequence of individual images and can be interrupted by the operator.
The images are displayed for the rover operator as they arrive and are also logged to disk. Mosaics of images taken at a single rover position can be merged together and printed as a seamless image annotated with azimuth and elevation. Through a pipeline of processing algorithms, high resolution stereo images can be turned into surface models overlaid with corresponding texture images.

\subsection{Robust communication}

The science images and sequences of images from Nomad must be reliably sent from the robot to one or more sites. Because the communication path is quite involved and subject to unpredictable losses in bandwidth, a novel communication scheme has been developed to provide reliable transmission of data through unreliable channels to multiple real-time display stations and logging computers.

The data communications between processors on-board Nomad and workstations was performed using the NDDS package. NDDS applies producer/consumer model and uses connectionless UDP packets for it's underlying data transport. With UDP there are no delivery guarantees but no acknowledgment delays. NDDS has an internal limit of 8 kilobytes bytes in a message, but because images are larger than this, they must be broken up into 8 kilobyte packets for transfer. We implemented a simple scheme for breaking images into packets with identifying header information.

To improve the bandwidth utilization, an algorithm was designed to stream packets out at a specified data rate and then use a protocol for requesting resends of missed packets. The image header includes the packet numbers that will make up the image. Whenever a gap in the packet sequence is detected, the missing packets are requested by the receiver. The image packet also contains the identifier of the image header it corresponds to. If a packet has an identifier that has not been received, a request is sent for the missing header.

If multiple receivers miss different packets or headers (due to ethernet collisions, processor saturation, or satellite interruptions), each one can request the packets that they missed. If a receiver receives a packet twice, it ignores the second copy. If the sender receives multiple requests for a packet resend within a time-out, it ignores the extra requests.

The image communication protocol has been built into the Virtual Dashboard which progressively displays images as they arrive.

The science imaging system needs to send sequences of images quickly in order to transfer image mosaics. This means that the resends on one image may not be complete before the next image transfer starts. Losses in communication can last for seconds or even minutes. Most messages sent during a communication drop out are lost. When the link is reestablished, all available bandwidth is used to resend missed image packets. Because of this change in available bandwidth for new image transmission, there must be a way to slow down an resending to avoid saturation and additional packet loss.

To avoid overflows, any receiver sends back the number of seconds since the input buffers where cleared. This is used to throttle back its sending rate. Due to the variable latencies in the real system, it is difficult to analyze or predict the 
changes in bandwidth. The timing and scale factors used were determined from field refinement and seem to recover from link losses of up to several minutes.

This multi-destination, reliable data transmission performed well under harsh, real world conditions. The science imager transferred over 400 megabytes of images over heavily-loaded radio, satellite, wide, and local area links.

This protocol could be used with multi-cast UDP packets to further improve performance when supplying a large number of receivers on a local area network. For high image rates, retransmissions could be suppressed if timeliness is more important than accuracy.

\subsection{Modeling from science images}

The use of photorealistic terrain models for telerobotic applications allows scientists and engineers to learn more and faster about the remote location by allowing them to virtually explore the terrain. For the Nomad project the science imaging system produced stereo pairs that were used to build models of the surroundings, shown in Figure 7. These terrain

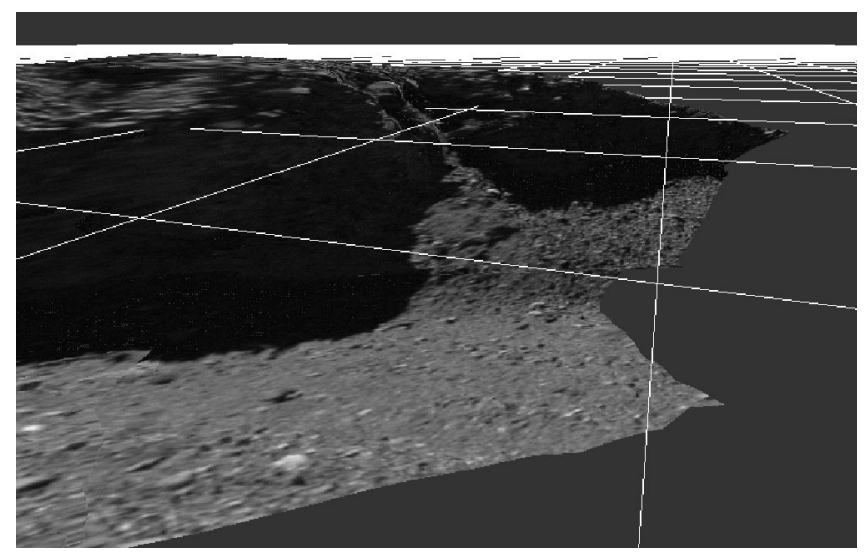

Figure 7: Terrain model built from 12 stereo pairs (including Figure 6) with images overlaid

models helped scientists define morphology of rocks, outcrops, washes and other surface features. They were also useful in determining trafficability. Another useful feature is the ability to produce a plan view of the rover surroundings. This type of view was used as a map to determine feature distribution and alignment, to estimate distances, and to plan paths.

The Nomad mission clearly showed the potential of telerobotics with on-line photorealistic model generation. During the trek, use of these models was limited because of the amount of data (thus the time) required to build models. Future implementations might combine terrain models with panospheric imagery for surface texture.

\section{Science Field Experiments}

The objectives for these science field experiments were to:

- Provide realistic desert experience for remote operators through high-quality imagery and a virtual environment interfaces

- Evaluate near-term planetary missions (to the Moon, Mars, and Antarctica) by training scientists, evaluating control environments appropriateness, developing and evaluating exploration strategies, and evaluating interaction in the science teams

- Evaluate the importance of various imaging techniques: panospheric imaging, foveal-resolution stereo imaging, image mosaicing, and textured terrain models

- Understand the reasons for correct and incorrect scientific interpretation by collecting ground-truth and carefully evaluating scientists' procedures/protocols

\subsection{Simulated Exploration Missions}

Using Nomad, our Virtual Dashboard and Telepresence interfaces, and the Science Imaging system, we conducted simulations of remote operations on Mars, the Moon and the Antarctic.

Two Mars mission simulations provided training for site characterization and sample caching operations. The site characterization exercise, in which scientists tried to correctly characterize the climate, geology and evidence of past life at a landing site, was conducted without panospheric or aerial imagery, in analog to the Mars Pathfinder mission. Scientists worked collaboratively to analyze images from the science cameras, resulting in a slow but thorough examination of the site. The sample caching exercise enabled all available imagery and resulted in nearly four times the area covered with a number of distinct rock types selected as samples.

In the Lunar mission simulation, planetary scientists from NASA and the USGS attempted to perform "geology-onthe-fly", in which they assessed trafficability and surveyed gross geology while keeping the rover in motion $75 \%$ of the time. This mode of operation is appropriate for long-distance exploration or for traverse between sites of scientific interest. In a record for remote exploration, Nomad traversed 1.3 kilometers and examined 10 science sites. During this test scientists also made the surprising discovery of a Jurassic fossil bed.

For the Antarctic test, we wished to evaluate the feasibility of searching visually and magnetically for meteorites with a remotely controlled robot. On-site geologists prepared a $100 \mathrm{~m}-\mathrm{by}-5 \mathrm{~m}$ area test area with surface and buried meteorites. Nomad made a patterned search, while remote geologists looked for indicative rock features. Of three visible meteorites geologists correctly identified one meteorite (and correctly rejected two meteorite-looking rocks). While searching with visual and magnetic sensors, they found that the readily identifiable magnetic signature helped to localize iron meteorites and significantly improved chance of discovery (three meteorites were found.)

Lastly, we conducted an experiment to determine the usefulness of the panospheric camera when operating with time delay. With a time-delay of 15 minutes (average for Mars), and both with and without panospheric imagery we performed the same task: approach a science site, image sufficient features to provide an interpretation, and repeat. With the panospheric imagery, fewer uninformative images were taken and twice as much area was examined. 


\subsection{Results and Discoveries}

Initial indications of the field experiment are that the ability to continually see all around the robot provides scientists with a sense of position and orientation at the remote site that has been lacking in previous field experiments and substantially benefits and accelerates site exploration. Panospheric imagery clearly improves efficiency. It enables assess the gross geology and quickly focus on key indicators. This has benefit when operating with a time delay.

Interesting too, was the observation that a time-delay itself improves efficiency because it tends to slow the action down leading to more thoughtful actions and less hurry to keep up with the pace of the exploration.

In previous field experiments [Thomas, 1995], we learned the importance of panoramic images to localizing the robot, understanding the surroundings and planning traverses. Now, the display of panospheric imagery fulfills the need for panoramic context and adds the speed and ease of the Telepresent Interface.

The science imaging system allowed better scientific exploration and interpretation of the remote geology than previous camera systems. This is attributed to the threefold increase in resolution and the readily available color. Although the color registration of the cameras was never calibrated, color clearly revealed many geologic features that were not obvious in monochrome images. Accuracy of geologic interpretations substantially improved over previous experiments with high-resolution color stereo imaging as a crucial factor.

The wide field-of-view monochrome cameras were also valuable. They were often used to take mosaics of the an area for geologic survey, structural identification, and site context. They were used for initial science investigation and to aim the high resolution cameras. These cameras were also valuable for examining obstacles to locomotion because they have a higher resolution than the panospheric camera.

Image mosaics and 3-D terrains should be more readily available, as this would further improve the exploration of the geology and the ability to navigate difficult terrain features. Tools that integrate the image and vehicle state databases would aid landmark tracking as well as the analysis of the geology of the region.

While noting that the demands of remote geology require practice, geologists concurred that even without a full suite of tools for geologic exploration (like a rock hammer and a hand lens), useful and accurate analysis can be performed remotely. For example, Nomad's high-resolution color stereo cameras revealed a previously unknown rock outcrop dating from the Jurassic era (Figure 8). Geologists identified the outcrop as a target of interest and navigated to it using panospheric images. Close inspection of several exposed rocks indicated fossilized material. Later analysis did not confirm these specific fossils but the rock outcrop was correctly characterized as a fossil bed. This is the first time geologists using a robot surrogate have made such a discovery, something that was widely thought to be too difficult to do remotely. This has important implications to future planetary exploration.

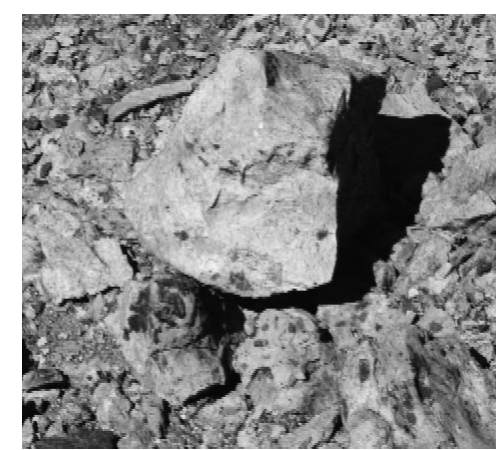

Figure 8: Image from Nomad of a Jurassic-era fossil bed.

\section{Acknowledgments}

The Nomad project was led by Carnegie Mellon University where Nomad was designed and built.We thank Dr. Red Whittaker, Eric Rollins, Mark Maimone, John Murphy, Mark Sibenac, Deepak Bapna, Jim Teza, Mike Parris, Ben Shamah, and Alex Foessel for enabling and encouraging our participation in the project.

We thank and acknowledge Dr. Geb Thomas of the University of Iowa GROK Lab for development of the Telepresence Interface.

The science mission simulations were designed and organized by Dr. Nathalie Cabrol of NASA Ames. We greatly appreciate her efforts and those of the science team members: Guillermo Chong, James Dohm, George Dunfield, Virginia Gulick, Ragnhild Landheim, Pascal Lee, Rendy

Keaten, Ted Roush, Carol Stoker, and Aaron Zent

We thank the entire Intelligent Mechanisms group for their efforts in support of the Atacama Desert Trek.

This work was supported by the NASA Space Telerobotics Program, David Lavery, Program Manager.

\section{References}

[Christian, 1997] Daniel Christian, David Wettergreen, Maria Bualat, et. al. Field Experiments with the Ames Marsokhod Rover. In Proceedings of the 1997 Field and Service Robotics Conference, Canberra, Australia, December 1997.

[Piguet, 1995] Laurent Piguet, Terry Fong, Butler Hine, et. al. VEVI: A Virtual Reality Tool for Planetary Exploration. In Proceedings of Virtual Reality World, Stuttgart, Germany, February 1995.

[RTI, 1997] Real-time Innovations. http://www.rti.com/ndds

[Summus, 1997] Summus Inc. http://www.summus.com

[Thomas, 1995] Hans Thomas, Butler Hine, and John Garvey. Kilauea: A Terrestrial Analog for Studying the Use of Rovers in Planetary Science. In Proceedings of the Conference of the Space Studies Institute, Princeton, New Jersey, May 1995.

[Whittaker, 1997] Red Whittaker, Deepak Bapna, Mark Maimone, and Eric Rollins. Atacama Desert Trek: A Planetary Analog Field Experiment In Proceedings of the International Symposium on Artificial Intelligence and Automation for Space, Tokyo, Japan, July 1997 